\title{
Oclusão de ramo da veia central da retina
}

\author{
Retinal vein branch occlusion
}

Alexandre Antonio Marques Rosa ${ }^{1}$

Trabalho realizado no Departamento de Oftalmologia do Hospital das Clínicas da Faculdade de Medicina da Universidade de São Paulo - USP.

${ }^{1}$ Pós-Graduando Nível Doutorado pela Faculdade de Medicina da Universidade de São Paulo - USP e membro do Departamento de Retina e Vítreo do Hospital das Clínicas da Faculdade de Medicina da Universidade de São Paulo - USP.

Endereço para correspondência: Rua Afonso de Freitas, 66/51 - São Paulo (SP) CEP 04006-050

E-mail: alexandre_ros@hotmail.com

Recebido para análise em 29.05.2003

Versão revisada recebida em 12.08.2003

Aprovação em 27.08.2003

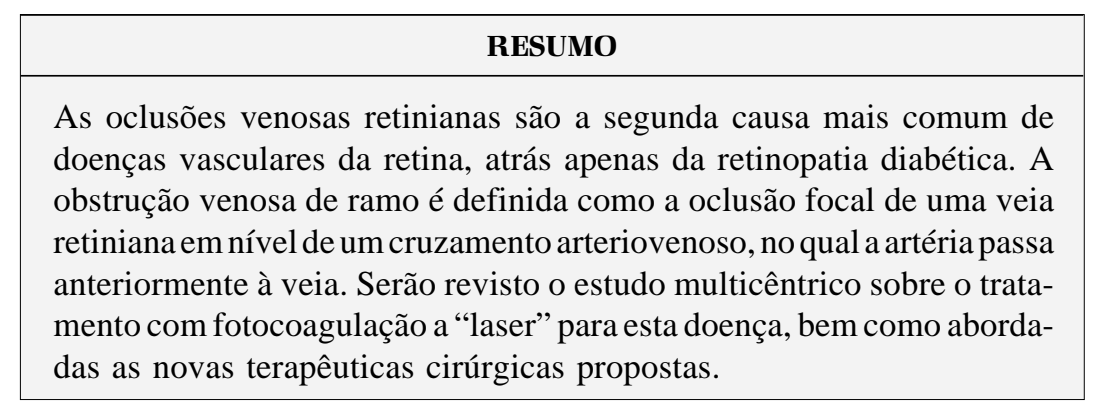

Descritores: Oclusão da veia retiniam; Veia retiniana/patologia; Fotocoagulação; Vitrectomia

\section{INTRODUÇÃO}

As oclusões venosas são a segunda causa mais comum de doenças vasculares da retina, atrás apenas da retinopatia diabética ${ }^{(1)}$.

A obstrução venosa de ramo é definida como a oclusão focal de uma veia retiniana ao nível de um cruzamento arteriovenoso, onde a artéria passa anteriormente à veia ${ }^{(2)}$ (Figura 1). É uma afecção quase sempre de início súbito, na qual o paciente apresenta visão borrada ou defeito de campo visual e hemorragias intra-retinianas distribuídas setorialmente. Afeta indivíduos de ambos os sexos, sendo mais freqüente entre 60 e 70 anos de idade $^{(3)}$.

Geralmente, as hemorragias intra-retinianas são menos graves se a oclusão é incompleta; o inverso é verdadeiro nas obstruções completas com resultante falta de perfusão capilar.

A localização da interrupção do fluxo venoso determina a distribuição das hemorragias intra-retinianas. Se a obstrução está situada próxima ao disco óptico, dois quadrantes do fundo podem ser atingidos (oclusões hemisféricas); no entanto se a oclusão é mais periférica, um quadrante, ou menos, pode estar envolvido. Quando o bloqueio é periférico às tributárias que drenam a mácula, pode não haver diminuição da visão ${ }^{(2)}$.

Zhao et al. examinando 106 olhos (104 pacientes) demonstraram que as oclusões ocorreram ao nível de um cruzamento arteriovenoso em $100 \%$ dos casos, e em $99 \%$ dos pacientes a artéria estava anterior a veia ${ }^{(4)}$.

$\mathrm{O}$ aspecto anatômico dos cruzamentos arteriovenosos - (a) a artéria e a veia apresentam uma bainha adventícia comum, (b) o lúmen venoso diminuído promove um turbilhonamento do fluxo sangüíneo, com conseqüente (c) dano endotelial, formação de trombo e, por fim, oclusão do vaso associado às alterações de esclerose arteriolar (aumento da rigidez) pode explicar a aparente vulnerabilidade destes locais à oclusão venosa.

Frangieh et al. demonstraram, através de estudos histopatológicos, a presença de trombos no local da oclusão em oito pacientes com obstrução venosa retiniana, com conseqüente esclerose moderada a grave em quase todas as arteríolas envolvidas ${ }^{(5)}$. 


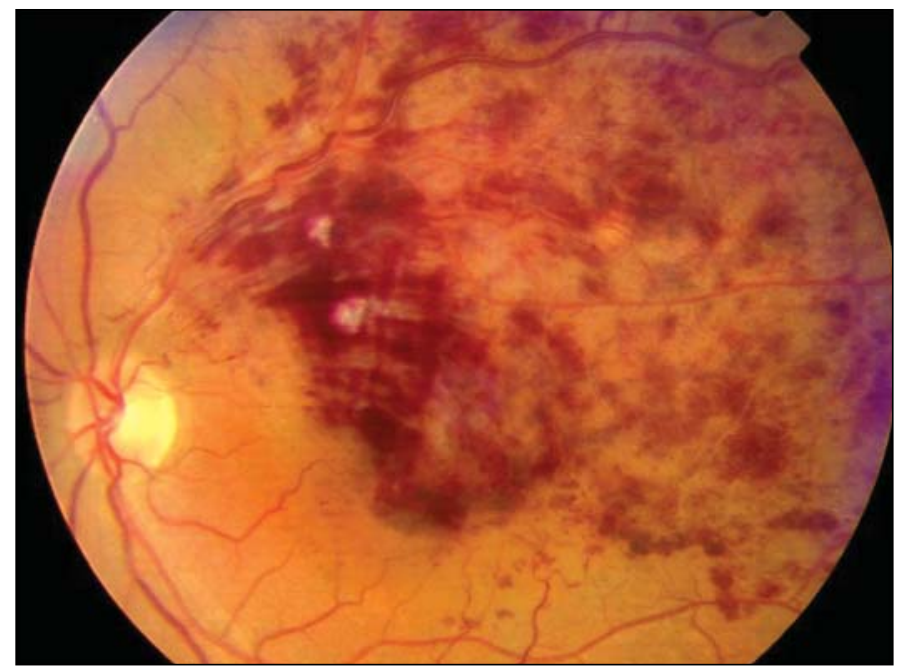

Figura 1 - Oclusão do ramo temporal superior da veia central da retina

As oclusões de ramo da veia central da retina ocorrem no setor temporal em $98 \%$ dos casos, e $66 \%$ destes acometem a arcada temporal superior ${ }^{(4)}$. As possíveis razões para o maior acometimento deste setor seriam: (1) Diminuição da acuidade visual por envolvimento da mácula e (2) maior número de cruzamentos no setor temporal superior ${ }^{(4)}$.

Várias condições sistêmicas têm sido relatadas como associadas à oclusão de ramo venoso da retina. A associação com glaucoma crônico de ângulo aberto ${ }^{(6-7)}$ não está tão bem estabelecida quanto à com a obstrução de via central da retina ${ }^{(8)}$. Alguns estudos têm demonstrado uma maior prevalência de pressão intra-ocular elevada em olhos com obstrução venosa de ramo ${ }^{(3,9)}$. A hipertensão arterial sistêmica é um fator de risco particularmente importante ${ }^{(6,8,10)}$. Em um estudo caso-controle examinando 270 pacientes com obstrução venosa, 51\% dos indivíduos eram hipertensos ${ }^{(3)}$. Outros fatores de risco identificados foram: alto índice de massa corporal, história de diabetes melito, história de doença cardiovascular, anormalidades no ECG, histórico de hipertensão ocular e altos níveis séricos de triglicérides e $\alpha_{2}$-globulina ${ }^{(3)}$.

A fotocoagulação a "laser" continua sendo o tratamento mais aceito para esta doença. O "Branch Vein Occlusion Study”(1,11) foi um estudo clínico, multicêntrico e aleatorizado, que examinou 401 olhos, entre julho de 1977 até fevereiro de 1984, com um tempo de seguimento médio de 3,1 anos, com o propósito de responder a 3 questões fundamentais:

(1) Pode a fotocoagulação impedir o desenvolvimento de neovascularização?

(2) Pode a fotocoagulação prevenir a hemorragia vítrea?

(3) Pode a fotocoagulação melhorar a AV em olhos com edema macular e acuidade visual igual ou pior que 0,5 ?

Em tal estudo, os pacientes foram divididos em quatro grupos:

- Grupo I (olhos de risco para desenvolverem neovascularização) com as seguintes características:

1. Entre três e dezoito meses de história.
2. Pelo menos cinco diâmetros de papila de área retiniana acometida.

3. Ausência de hemorragias retinianas para permitir adequada fotocoagulação.

4. Ausência de retinopatia diabética.

5. Ausência de outras doenças oculares que pudesse causar baixa de visão (pacientes com retinopatia diabética ou outras doenças oculares foram excluídos do estudo).

A avaliação do paciente na fase aguda (três primeiros meses) está bastante prejudicada, pois freqüentemente há hemorragias intrarretinianas extensas envolvendo a mácula e o centro da fóvea bloqueando a visão dos vasos.

Trezentos e dezenove olhos foram incluídos neste grupo, com seguimento médio de 3,7 anos. Dos 160 olhos randomizados para receberem tratamento com fotocoagulação a "laser", 12\% desenvolveram neovascularização. No grupo controle, quase o dobro (22\% dos 159 olhos) desenvolveram neovasos ${ }^{(11)}$.

- Grupo II (olhos de risco para desenvolverem hemorragia vítrea)

1. Entre três e dezoito meses de história.

2. Presença de neovascularização periférica e/ou de disco.

3. Ausência de hemorragias retinianas para permitir adequada fotocoagulação.

4. Ausência de retinopatia diabética.

5. Ausência de outras doenças oculares que pudesse causar baixa de visão.

Dos 82 olhos selecionados para este grupo, cujo seguimento médio foi de 2,8 anos, 41 foram aleatorizados para receberem tratamento e 41 para não receberem. Dos olhos tratados, 29\% desenvolveram hemorragia vítrea, enquanto que $61 \%$ dos olhos não tratados apresentaram sangramento para a cavidade vítrea ${ }^{(12)}$. O objetivo da fotocoagulação era obter marcas de intensidade média (200 a $500 \mu$ de diâmetro), eqüidistantes entre si, cobrindo todo o seguimento envolvido e estendendo-se a, no máximo, dois diâmetros de papila do centro da fóvea ${ }^{(11)}$.

- Grupo X (Olhos de alto risco para desenvolverem neovascularização)

1. Entre três e dezoito meses de história.

2. Pelo menos cinco diâmetros de papila de área de não perfusão capilar na angiofluoresceinografia retiniana.

3. Ausência de outras doenças oculares que pudesse causar baixa de visão.

Na verdade, este grupo foi criado para manter uma reserva de casos que posteriormente foram incluídos no grupo II. Porém, uma das conclusões mais interessantes deste estudo foi proveniente justamente deste grupo. De todos os olhos com alto risco para desenvolver neovascularização, menos da metade (41\%) de fato desenvolveram neovasos ${ }^{(11)}$.

Portanto, não se justifica a realização de fotocoagulação em todos os pacientes, pois mesmo que selecionássemos apenas casos de maior risco, mais da metade destes nunca desenvolverão neovascularização. Por esta razão, recomenda-se que a fotocoagulação seja aplicada apenas quando a neovascularização é vista. 
A neovascularização de disco ou retiniana, ou ambas, podem desenvolver-se a qualquer tempo dentro dos três primeiros anos após a obstrução, sendo mais comum nos primeiros seis a doze meses ${ }^{(12)}$.

Neovascularização de íris é uma complicação rara da oclusão venosa de ramo, parece que pacientes com diabetes (com ou sem retinopatia) têm um risco aumentado ${ }^{(13)}$.

A neovascularização é particularmente difícil de reconhecer em oclusão venosa de ramo porque os colaterais que freqüentemente se desenvolvem assemelham-se a neovasos. Presumese que os colaterais apareçam a partir de capilares pré-existentes, formando canais entre as veias ao redor do local do bloqueio, ao longo da rafe temporal e em outros locais para desviar o fluxo a partir do segmento retiniano comprometido.

- Grupo III (olhos de risco para perda visual devido edema macular)

1. Entre 3 e 18 meses de história

2. Acuidade visual corrigida igual a ou pior que 0,5 .

3. Edema macular com vazamento angiográfico (Figura 2).

4. Ausência de hemorragias retinianas para permitir adequada avaliação da angiofluoresceinografia retiniana e realização da fotocoagulação.

5. Ausência de hemorragia diretamente sobre a fóvea.

6. Ausência de outras doenças oculares que pudesse causar baixa de visão.

Na verdade, estes acabaram se tornando os critérios para tratamento do edema macular relacionado com as oclusões venosas.

Foram incluídos 78 olhos neste grupo, 43 aleatorizados para receberem tratamento, e o restante (35 olhos) não. Após um seguimento médio de três anos, $60 \%$ dos olhos submetidos a fotocoagulação a "laser" apresentavam acuidade visual igual a ou melhor que 0,5 . Já no grupo controle, apenas 35\% apresentavam o mesmo nível de acuidade visual ${ }^{(1)}$. A fotocoagulação era realizada sobre a área de vazamento angiográfico, estendendo-se da margem da zona avascular da fóvea até a arcada vascular temporal.

Antes de realizar a fotocoagulação, é importante obter uma angiografia de ótima qualidade da região macular para demonstrar que o edema envolve o centro da fóvea e que não há uma grande quantidade de exclusão capilar adjacente a zona avascular da fóvea que explicaria a perda de visão.

Quando o exame clínico mostra um espessamento macular ou de aspecto cistóide nos primeiros seis meses após a oclusão, e há pouco ou nenhum vazamento no angiograma, a isquemia macular pode ser a causa do edema.

Finkelstein demonstrou que $91 \%$ dos pacientes com edema macular isquêmico, ou seja, sem vazamento angiográfico, apresentam melhora visual espontânea quando comparados àqueles com edema angiográfico $(29 \%)^{(13)}$. Portanto, pacientes com espessamento macular e ausência de perfusão apresentariam melhor prognóstico visual.

Na literatura neurológica, é amplamente relatada e reconhecida clinicamente a existência de dois tipos de edema cerebral, denominados vasogênico e citotóxico ${ }^{(14-15)}$. O edema cerebral vasogênico ocorreria por um extravasamento de água e proteínas plasmáticas dos vasos cerebrais. Por outro lado, a sua variante chamada de edema citotóxico refere-se a uma alteração celular no parênquima cerebral por hipertonicidade intracelular, seguida de hipertonicidade extracelular, que causaria o edema. Um fenômeno semelhante na retina isquêmica poderia ser responsável pelo edema transitório(13).

Nos últimos anos, uma nova modalidade terapêutica foi proposta para o tratamento de pacientes com oclusão de ramo da veia central da retina - particularmente aqueles com acuidade visual inicial muito baixa - que consistia na realização de vitrectomia posterior via "pars plana” e disssecção da bainha adventícia do cruzamento arteriovenoso responsável pela trombose (Figura 3). O objetivo era aliviar o fator compressivo e acelerar a recanalização do segmento acometido.

A técnica foi originalmente proposta por Osterloh e Charles em 1988, onde eles relatam o caso de um paciente que oito meses após ter sido submetido à cirurgia de vitrectomia posterior com "bainhotomia” apresentou uma melhora da acuidade

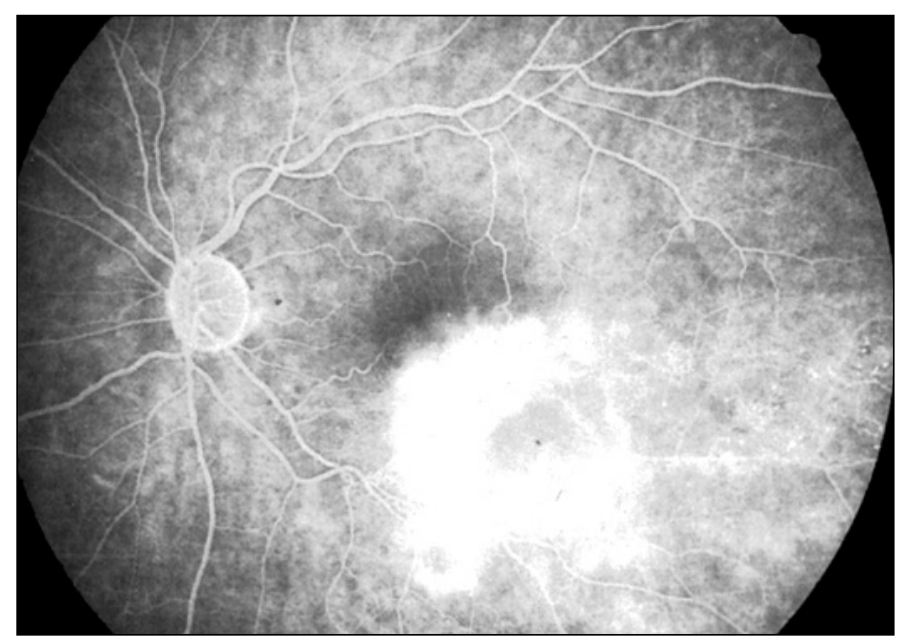

Figura 2 - Edema macular com vazamento na angiofluoresceinografia

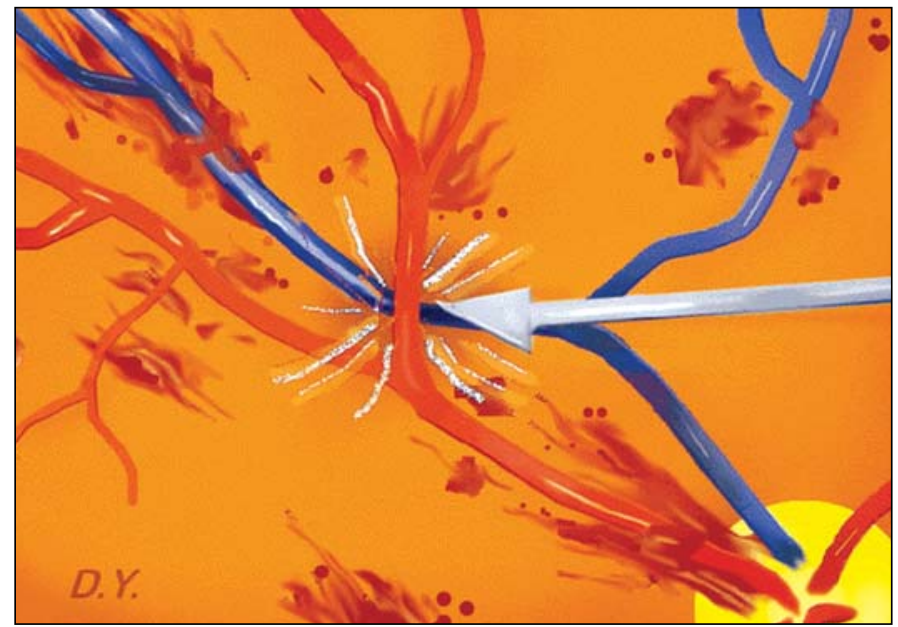

Figura 3 - Dissecção da bainha adventícia ao nível do cruzamento arteriovenoso acometido 
visual de 0,1 para 0,8 . Vasos mais próximos do nervo óptico seriam mais resistentes a manipulação cirúrgica ${ }^{(16)}$.

Opremcak e Bruce fizeram um estudo prospectivo, com seguimento médio de cinco meses, de 15 olhos submetidos à cirurgia. Os pacientes apresentavam: AV 0,3 ou pior devido edema macular e extensas hemorragias envolvendo a fóvea (46\% dos casos apresentavam isquemia macular) e duração dos sintomas entre um e doze meses (média 3,3 meses). Todos os olhos apresentaram melhora clínica, com resolução das hemorragias retinianas e do edema de mácula. A acuidade visual ficou igual ou melhor em $80 \%$ dos casos (12 olhos) ${ }^{(17)}$.

No entanto, o papel da cirurgia vítreo-retiniana nos casos de edema macular associado às oclusões vasculares ainda necessita ser determinado, visto que existe a possibilidade de melhora espontânea em percentual significativo de casos. No "Branch Vein Occlusion Study"(1), 35\% dos olhos não tratados alcançaram uma acuidade visual melhor que ou igual a 0,5 após três anos de seguimento. Além disso, pacientes com edema macular isquêmico apresentam melhor prognóstico ${ }^{(13)}$.

Takahashi et al. avaliando 58 olhos, determinaram a relação entre o edema macular em pacientes com oclusão de ramo da veia central da retina e a condição do vítreo posterior. Os pacientes foram divididos em: Grupo I (com até 64 anos idade) e Grupo II (com 65 ou mais anos). Edema macular foi observado em $67 \%$ dos casos (39 olhos). Foi encontrada uma adesão vítreo-macular em $81 \%$ dos olhos do grupo I, dos quais $77 \%$ tinham edema. No grupo II, $45 \%$ dos olhos possuíam adesão do vítreo sobre a mácula, e destes, 93\% apresentavam edema ${ }^{(18)}$.

A aderência do vítreo posterior talvez exerça um papel importante na persistência do edema macular nestes pacientes ${ }^{(18)}$. Portanto, vitrectomia posterior associada ao descolamento da hialóide posterior poderiam afetar a história natural da doença, retardando a neovascularização, bem como prevenindo edema macular persistente.

A descompressão cirúrgica da oclusão vascular é um procedimento exeqüível. No entanto, até que um estudo clínico multicêntrico seja realizado, esta técnica não pode ser recomendada como um procedimento rotineiro.

\section{ABSTRACT}

Retinal venous occlusions are the second most common retinal vascular diseases, behind diabetic retinopathy. The retinal vein branch occlusion is defined as a retinal vein focal occlusion at the level of an arteriovenous crossing, where the artery lies anterior to vein. The clinical trial with laser photocoagulation for this disease, as well as new proposed surgical therapeutic techniques will be reviewed.

Keywords: Retinal vein occlusion; Retinal vein/pathology; Light coagulation; Vitrectomy

\section{REFERÊNCIAS}

1. Argon laser photocoagulation for macular edema in branch vein occlusion. The Branch Vein Occlusion Study Group. Am J Ophthalmol 1984;98:271-82.

2. Gutman FA, Zegarra $\mathrm{H}$. The natural course of temporal retinal branch vein occlusion. Trans Am Acad Ophthalmol Otolaryngol 1974;78:OP178-92.

3. Risk factors for branch retinal vein occlusion. The Eye Disease Case-control Study Group. Am J Ophthalmol 1993;116:286-96.

4. Zhao J, Sastry SM, Sperduto RD, Chew EY, Remaley NA. Arteriovenous crossing patterns in branch retinal vein occlusion. The Eye Disease CaseControl Study Group. Ophthalmology 1993;100:423-8.

5. Frangieh GT, Green WR, Barraquer-Somers E, Finkelstein D. Histopathologic study of nine branch retinal vein occlusions. Arch Ophthalmol 1982;100: 1132-40.

6. Johnston RL, Brucker AJ, Steinmann W, Hoffman ME, Holmes JH. Risk factors of branch retinal vein occlusion. Arch Ophthalmol 1985;103:1831-2.

7. Blankenship GW, Okun E. Retinal tributary vein occlusion. History and management by photocoagulation. Arch Ophthalmol 1973;89:363-8.

8. Orth DH, Patz A. Retinal branch vein occlusion. Surv Ophthalmol 1978; 22:357-76.

9. Frucht J, Shapiro A, Merin S. Intraocular pressure in retinal vein occlusion. Br J Ophthalmol 1984;68:26-8.

10. Appiah AP, Trempe CL. Differences in contributory factors among hemicentral, central, and branch retinal vein occlusions. Ophthalmology 1989;96:364-6.

11. Argon laser scatter photocoagulation for prevention of neovascularization and vitreous hemorrhage in branch vein occlusion. A randomized clinical trial. Branch Vein Occlusion Study Group. Arch Ophthalmol 1986;104:34-41.

12. Hayreh SS, Rojas P, Podhajsky P, Montague P, Woolson RF. Ocular neovascularization with retinal vascular occlusion. III. Incidence of ocular neovascularization with retinal vein occlusion. Ophthalmology 1983;90:488-506.

13. Finkelstein D. Ischemic macular edema. Recognition and favorable natural history in branch vein occlusion. Arch Ophthalmol 1992;110:1427-34.

14. Klatzo I. Presidental address. Neuropathological aspects of brain edema. J Neuropathol Exp Neurol 1967;26:1-14.

15. O’Brien MD. Ischemic cerebral edema. A review. Stroke 1979;10:623-8.

16. Osterloh MD, Charles S. Surgical decompression of branch retinal vein occlusions. Arch Ophthalmol 1988;106:1469-71.

17. Opremcak EM, Bruce RA. Surgical decompression of branch retinal vein occlusion via arteriovenous crossing sheathotomy: a prospective review of 15 cases. Retina 1999;19:1-5.

18. Takahashi MK, Hikichi T, Akiba J, Yoshida A, Trempe CL. Role of the vitreous and macular edema in branch retinal vein occlusion. Ophthalmic Surg Lasers 1997;28:294-9.

\section{ABO ELETRÔNICO \\ Novo site \\ Acesso: http://www.abonet.com.br}

